\title{
Star Players in the NBA - Decoys or Game-Changers?
}

\author{
Yangmufeng Liu \\ Correspondence: Yangmufeng Liu, Hwa Chong Institution (College), 661 Bukit Timah Rd, Singapore 269734. Tel: 65- \\ 8453-8592. E-mail: 1623221@student.hci.edu.sg
}

Received: December 30, 2020 Accepted: March 12, 2021 Online Published: March 15, 2021

doi:10.5539/jmr.v13n2p40

URL: https://doi.org/10.5539/jmr.v13n2p40

\begin{abstract}
In the National Basketball Association (NBA), when an attacking team is trailing by a small margin in the last seconds of a game, the coach has to make a critical decision: should he or she design a play for the star player or a non-star player? In this strategic situation, the offensive team can choose to let either the star or the non-star to shoot the ball while the defensive team can either double-team the star player or play conventional one-on-one defence. For the purpose of this research, star players are divided into two types - perimeter and interior. Given different point deficits, an equilibrium point is obtained and comparative statics are computed to analyze how the equilibrium will change given a change in a player's shot percentage. It is found that the offensive team should play the star player at most 3 out of 10 times in all scenarios.
\end{abstract}

Keywords: star players, basketball, game theory, National Basketball Association

\section{Introduction}

In the waning seconds of Game 6 of the 1993 NBA Finals, the defending champion Chicago Bulls faced a crucial situation where they were trailing by 2 points with 14.4 seconds left. During the final time out, Michael Jordan, aware that he may be double-teamed in the following possession, told his teammate John Paxson to prepare for a 3-point shot. Jordan successfully predicted the double-team and passed the ball to Paxson on time, who hit the game-winning shot and helped the franchise secure its first three-peat.

In the NBA, victory is desired not only because of the glory it brings but also the monetary gains. The 2019 NBA champion Toronto Raptors took home around \$3.6 million from the \$22 million "player's pool" that is divided among all playoff teams (Carter. 2019). Players' and coaches' contracts often include bonus clauses that grant them more money based on how many games they win. On the other hand, when a team loses games, reputations and job prospects are at stake. After the 2017-2018 season, 9 teams saw coaching changes before the start of the new season (McCarney, 2018). Therefore, sound decision-making is crucial in the competitive NBA, which makes it an ideal environment to apply and test game theory principles.

The end of a game is usually when critical decisions are made that determine its outcome. When the scores are close, the two teams are presented with various offensive and defensive strategies, such as whether the trailing team should attempt a two-point or a three-point shot, or whether the leading team should intentionally foul when they are defending. In previous research, much attention has been devoted to team strategies from a collective point of view, irrespective of the type of players on the team. Less studied is how the traits and abilities of individual players can affect the strategies that the team opts for. The coach of the trailing team is often confronted with a hard choice: designing a play for the star or a non-star player in the last possession? He might be tempted to put the ball into the star player's hands, but at the same time, the defensive team can always counter by double-teaming the star player, or playing conventional defense if the offensive team chooses a non-star player to shoot the ball. In this paper, game theory is utilised to evaluate specifically how the position and capability of individual players can influence the offensive and defensive strategies in different end-game scenarios.

A concise outline of this paper is as follows. Section 2 discusses previous research related to strategic play in the field of competitive sports, especially basketball. Section 3 presents the mathematical model of this strategic situation, including various parameters and assumptions made to simplify the game. Sections 4-7 proceed to analyze different scenarios and evaluate the results respectively. In Section 8, sensible data are used to compute equilibrium in different scenarios and the implication of the results is discussed. Finally, Section 9 concludes and discusses the direction for future research. 


\section{Literature Review}

Competitive sports, especially basketball, have been one of the most important fields to investigate game theory principles as teams or individuals adopt strategies to maximise their chances of winning.

Basketball is a strategy-intensive game, and many works have considered the optimal strategy. Charkrabarti and Dutta (2019) and Ashour et al. (2019) formulated basketball as a repeated game and adopted an algorithmic approach to compute the optimal offensive and defensive tactic options for specific teams based on expected points scored for different players. Skinner (2012) derived a function of shot quality considering the time left on the shot clock and used it to predict the shot percentage of a team under optimal conditions as a function of time. Its main conclusion is that NBA teams may be too reluctant to attempt a shot early in their possession. Other general works have centered around the decision-making process in basketball. $\mathrm{Ni}$ and McGarrity (2013) compared the quality of strategic choices by players and the coach and argued that coaches are better than players in making good strategic choices. In particular, coaches are better at assigning shots randomly so the defense cannot discover a pattern and react. Sindik and Vidak (2008) discussed how the extent of predictability of individual players in a team setting can affect the tactical performance of his or her team and the opponent team. In other sports, research has also been done to analyze strategic situations. For example, Palacios-Huerta (2003) considered the penalty kick situation in soccer and found a consistency between theoretical equilibrium derived from Minimax theorem and empirical choices made by players.

One of the most commonly studied situations is the offensive and defensive strategies in end-game scenarios with close scores. Ruminski (2012) modeled basketball as a zero-sum game and evaluated whether the defensive team should defend a two or three-point shot at the end of a game. Chow et al. (2020) more generally provided both sequential and simultaneous game analysis of mixed strategy Nash equilibrium when the offensive team is trailing by 2 or 3 points. However, the two aforementioned papers only considered the most general case but failed to factor in other possible situations like fouling and individual players' abilities.

One specific aspect that has been taken into account in analyzing end-game strategies is the fouling situation. In his paper, Ruminski also evaluated the most efficient way to foul throughout the game. He concluded that benching a player in foul trouble is more beneficial in early quarters than in the last quarter. A theoretical analysis of the fouling situation at the end of the game was presented in Annis (2006) in which two strategies, committing a foul and not fouling, are compared. It was concluded that the defensive team leading by three points is always better off if they are to foul and let the offensive team attempt two free throws. This conclusion challenges the belief of the majority of basketball coaches that committing a foul is usually less desirable than not doing so. This conclusion is supported by the statistical model presented in Kenter (2015). By modelling basketball as a combinatorial game, he considered when a team should start to foul given a point differential. He found that a team down by 5 points should foul when there are about 45 seconds left in the game, and that a team down by 8 should start to foul with roughly 90 seconds remaining. Generally, the paper suggested that trailing teams should begin to foul much earlier and more often; the leading team should foul earlier with larger leads and more often.

One issue in end-game situations that has not been addressed is how different players with varying abilities and roles can influence the strategies adopted by coaches. This paper will consider this question from the perspective of the offensive team and analyze the mixed strategy given different point differentials and types of players.

\section{Mathematical Modeling}

For the purpose of the discussion in this paper, we shall assume that each of the offensive and defensive team has two strategies each coach can adopt.

For the offensive team, the coach can instruct either the star player $(S)$ on the team or a specific non-star player $(N S)$ to take the shot. To enrich our discussion, star players are further classified into perimeter and interior players. There is not a set of exact criteria for a player to be qualified as a star; however, for the purpose of our discussion, it is assumed that a star player must generally fulfil the following conditions. Firstly, he must have one the highest average points per game in the team. Secondly, the star player's scoring ability must be consistent. This means that they player's scores must sustain over time, for example above 20 points nearly every game. Further assumptions made about the two types of players are introduced in the assumption section.

The defensive team can either double-team the star player $(D)$ or to employ normal one-on-one defense $(N D)$. The defensive team does not have the choice of doubling a non-star player on the opposing team as they do not have prior knowledge of which non-star player would take the shot should the offense chooses $N D$. 
We now proceed to define the notations used in the strategic situation in Table 1 .

Table 1. Notations

\begin{tabular}{cr}
\hline Notation & Meaning \\
\hline$P_{C S}$ & contested shot percentage of star player under double-team defense \\
\hline$P_{O S}$ & open shot percentage of non-star player \\
\hline$P_{S}$ & star player shooting percentage under normal defense \\
\hline$P_{N S}$ & non star player shooting percentage under normal defense \\
\hline$P_{1}$ & probability of offensive team playing $S$ \\
\hline$P_{2}$ & probability of defensive team playing $D$ \\
\hline
\end{tabular}

In order to properly analyze the strategic situation, several assumptions are made about the possible actions of each team and the possible events at the end of the game (Annis, 2006):

1. Perimeter star players will always attempt a three-point shot whereas interior players will always attempt a two-point shot.

2. The offensive team can score at most once in regulation.

3. The player chosen by the offense to shoot the ball must take the shot i.e. he cannot pass to other players.

Justification: In reality, all players will typically react to the given situation, and might choose to pass the ball under a double-team defense even if instructed to shoot by the coach. Nonetheless, for simplicity, we shall assume the most common case where the players execute the original play designed by the coach and do not pass the ball.

4. When the leading team chooses $D$ and the trailing team chooses $N S$, there will always be an open shot.

Justification: This implies that if the offensive team chooses $N S$, the particular player who takes the shot is not known to the defensive team. This assumption is commonly true in the NBA, since the defensive team may not have an incentive to double-team a non-star player at the expense of allowing the star to get an open shot.

5. The leading team will not score again in regulation.

6. If the game goes to overtime, each team will have a $50 \%$ chance of winning.

7. In the case of perimeter star players with non-star player shooting three-point shots, we shall assume $P_{O S}>P_{S}>$ $P_{N S}>P_{C S}$.

Justification: This would be the most sensible assumption since it reflects several common facts in a real basketball game. First, star players normally shoot better than non-star players. This offers the defensive team the incentive to doubleteam the star player as the contested shooting percentage is most likely the lowest. Moreover, this situation also ensures strategic play for the offense since an open shot by a non-star player will yield the highest possibility.

8. In the case of perimeter star players with non-star player shooting 2 pointers, we shall assume $P_{O S}>P_{N S}>P_{S}>P_{C S}$. Justification: This is sensible since it is easier to make 2-point shots than 3-point ones.

9. In the case of interior star player, we shall assume $P_{S}>P_{O S}>P_{C S}>P_{N S}$.

Justification: This is based on the observation that it is easier to score closer to the basket and star interior players facing defense tend to have higher shooting percentage around the paint area than open non-star players. This assumption illustrates that at areas closer to the basket, interior star players' advantage is more apparent in that they have a higher chance to score although they can only score 2 points.

In subsequent sections, cases are divided based on the type of star player on the team and the point difference in the game. The 4 cases analyzed are denoted as Case A (perimeter star player, down by 3 points), Case B (perimeter star player, down by 2 points), Case $\mathbf{C}$ (interior star player, down by 3 points) and Case $\mathbf{D}$ (interior star player, down by 2 points).

\section{Case A: Perimeter Star Player, Down by 3 Points}

In this section, it is assumed that the offensive team is trailing by 3 points with a perimeter star player on the team. According to assumption 2, it is only sensible for the coach to opt for a three-point shot regardless of the type of the player who takes the shot in the hope of tying the game and going to overtime. This scenario is widely seen in the NBA today since the popularization of three-point shots has caused many star players to consistently shoot 3-pointers at a high 


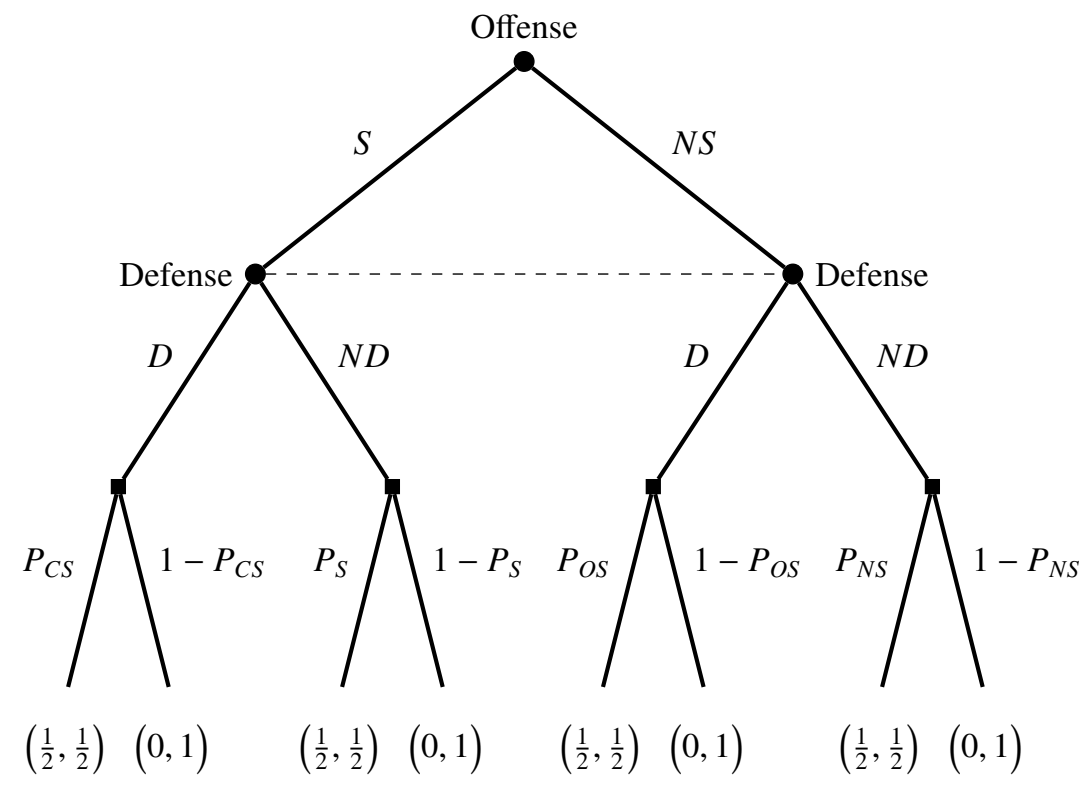

Figure 1. Game tree for Case A

shot percentage. Consider, for example, the 2019-2020 Houston Rockets. In an end-game situation, the coach Mike D'Antoni's first offensive choice would be James Harden, the star player on the team who has an average 3-point field goal made per game of 4.4, the highest in the league (NBA, 2020). However, this piece of information is also known to the opponent team's coach, who might choose to play double-team defense on Harden. Hence, D'Antoni also needs to consider letting a non-star player, say P.J. Tucker, who ranks the second in terms of 3-point field goal percentage on the team (37.3\%) (NBA, 2020) and has been consistently hitting open shots although he is not good at creating shot opportunities under tight defense.

The game tree (Figure 1) and table of expected payoff (Table 2) are presented below. In Figure 1, the dashed line in the game tree indicates an information set. In this strategic situation, the defensive team does not have prior knowledge of the strategy played by the offensive team.

Table 2. Expected payoff for Case A

\begin{tabular}{cccc|}
\multicolumn{4}{c}{ Offense } \\
\cline { 3 - 4 } Defense & $D$ & \multicolumn{1}{c}{$S$} \\
\cline { 3 - 4 } & $\left(1-\frac{1}{2} P_{C S}, \frac{1}{2} P_{C S}\right)$ & $\left(1-\frac{1}{2} P_{O S}, \frac{1}{2} P_{O S}\right)$ \\
\cline { 2 - 4 } & $N D$ & $\left(1-\frac{1}{2} P_{S}, \frac{1}{2} P_{S}\right)$ & $\left(1-\frac{1}{2} P_{N S}, \frac{1}{2} P_{N S}\right)$ \\
\cline { 2 - 3 } & &
\end{tabular}

\subsection{Analysis}

Assuming $P_{O S}>P_{S}>P_{N S}>P_{C S}$, there is no pure strategy equilibrium. To evaluate the mixed strategy Nash equilibrium, the expected payoff a team gets from the opponent playing a particular strategy will be computed.

At the equilibrium, the offense should mix between $S$ and $N S$ such that the expected payoff of the defense playing $D$ is equal to that of $N D$.

$$
\begin{aligned}
\mathbb{E}[D] & =\mathbb{E}[N D] \\
\left(1-\frac{1}{2} P_{C S}\right) P_{1}+\left(1-\frac{1}{2} P_{O S}\right)\left(1-P_{1}\right) & =\left(1-\frac{1}{2} P_{S}\right) P_{1}+\left(1-\frac{1}{2} P_{N S}\right)\left(1-P_{1}\right)
\end{aligned}
$$

After some algebraic manipulation and solving for $P_{1}$, we get: 


$$
P_{1}=\frac{P_{O S}-P_{N S}}{P_{S}+P_{O S}-P_{C S}-P_{N S}}
$$

$P_{1}$ represents the equilibrium probability that the offensive team should design a play for their star player. To see how changes in each of the parameters affect this value, we need to calculate comparative statics (i.e. partial derivatives of $P_{1}$ with respect to each of the exogenous parameters).

$$
\begin{gathered}
\frac{\partial P_{1}}{\partial P_{S}}=\frac{P_{N S}-P_{O S}}{\left(P_{S}+P_{O S}-P_{C S}-P_{N S}\right)^{2}}<0 \\
\frac{\partial P_{1}}{\partial P_{O S}}=\frac{P_{S}-P_{C S}}{\left(P_{S}+P_{O S}-P_{C S}-P_{N S}\right)^{2}}>0 \\
\frac{\partial P_{1}}{\partial P_{C S}}=\frac{P_{O S}-P_{N S}}{\left(P_{S}+P_{O S}-P_{C S}-P_{N S}\right)^{2}}>0 \\
\frac{\partial P_{1}}{\partial P_{N S}}=\frac{P_{C S}-P_{S}}{\left(P_{S}+P_{O S}-P_{C S}-P_{N S}\right)^{2}}<0
\end{gathered}
$$

Equations 2 and 5 suggest that when the offensive team's performance under normal defense improves, they should play the star player less. This result might be counter-intuitive. However, when the expected payoff for $N D$ increases, the offensive team will play $S$ more than $N S$ when the defensive team chooses $N D$ since $P_{S}$ is greater than $P_{N S}$. Hence the defense will counter by playing $D$ more. As a result, the offensive team will play $N S$ more to counter defense playing $D$, thus restoring the equilibrium.

Equations 3 and 4 suggest that when the offense improves their performance when facing double-team defense, they should play their star player more. This result can be explained by a similar analysis to the one provided in the previous paragraph.

Similarly, the defensive team should make the expected payoffs the offensive team receives from playing $S$ and $N S$ equal so that the offense is indifferent to playing $S$ or $N S$.

$$
\begin{aligned}
\mathbb{E}[S] & =\mathbb{E}[N S] \\
\frac{1}{2} P_{C S} P_{2}+\frac{1}{2} P_{S}\left(1-P_{2}\right) & =\frac{1}{2} P_{O S} P_{2}+\frac{1}{2} P_{N S}\left(1-P_{2}\right)
\end{aligned}
$$

Solving for $P_{2}$, we get:

$$
\begin{gathered}
P_{2}=\frac{P_{S}-P_{N S}}{P_{S}+P_{O S}-P_{C S}-P_{N S}} \\
\frac{\partial P_{2}}{\partial P_{S}}=\frac{P_{O S}-P_{C S}}{\left(P_{S}+P_{O S}-P_{C S}-P_{N S}\right)^{2}}>0 \\
\frac{\partial P_{2}}{\partial P_{O S}}=\frac{P_{N S}-P_{S}}{\left(P_{S}+P_{O S}-P_{C S}-P_{N S}\right)^{2}}<0 \\
\frac{\partial P_{2}}{\partial P_{C S}}=\frac{P_{S}-P_{N S}}{\left(P_{S}+P_{O S}-P_{C S}-P_{N S}\right)^{2}}>0 \\
\frac{\partial P_{2}}{\partial P_{N S}}=\frac{P_{C S}-P_{O S}}{\left(P_{S}+P_{O S}-P_{C S}-P_{N S}\right)^{2}}<0
\end{gathered}
$$

Equations 7 and 9 show that when the star player is better, defense should choose to double him more often. This result is expected since the defensive team has the incentive to double the star player more when he can make shots at a higher probability. 
Equations 8 and 10 show that when the opposing team has better non-star players, the defense should double the star player less, since the threat of non-star players becomes more significant.

\section{Case B: Perimeter Star Player, Down by 2 Points}

In this scenario, the offensive team has more choices as they can attempt either a 2-pointer or a 3-pointer. However, it should be noted that the restriction put on the star player is that he can only shoot a three pointer (perimeter star player). Therefore, this flexibility of choice of shot types is only applied to non-star players.

\subsection{Case B1: Non-star Players to Shoot Three-Point Shots}

We will first discuss the situation where the non-star player is instructed to shoot a 3-pointer. One classic instance is Game 6 of the 1993 NBA finals as mentioned in the introduction, when Michael Jordan dribbled into a double-team defense and made the pass to John Paxson, whose clutch 3-point shot helped the Bulls clinch the championship.

The game tree is presented in Appendix A. The table of expected payoff is shown in Table 3

Table 3. Expected payoff for Case B1

$$
\begin{array}{cc|c|c|}
\multicolumn{1}{c}{\text { Offense }} \\
\cline { 3 - 4 } \text { Defense } & D & \multicolumn{2}{c}{S} \\
\cline { 3 - 4 } & & \left(1-P_{C S}, P_{C S}\right) & \left(1-P_{O S}, P_{O S}\right) \\
\cline { 3 - 4 } & N D & \left(1-P_{S}, P_{S}\right) & \left(1-P_{N S}, P_{N S}\right) \\
\cline { 3 - 4 } & & &
\end{array}
$$

\subsubsection{Analysis}

The mixed strategy Nash equilibrium is the same as that of Case $\mathbf{A}$.

\subsection{Case B2: Non-Star Players to Shoot Two-Point Shots}

This situation occurs when the offensive team lacks reliable perimeter shooters on the team except for the star player. As a result, the offensive team coach would opt to "play safe" by settling for a closer 2-point shot. For example, although Trae Young of the Atlanta Hawks has a high three-point field goal made and percentage, the teams ranks the worst in terms of three-point field goal percentage at 33.4\% (NBA, 2020). Hence, the coach would prefer to let non-star players to shoot two-point field goals.

The game tree is presented in Appendix B. The table of expected payoff is shown in Table 4

Table 4. Expected payoff for Case B2

$$
\begin{array}{cc|c|c|}
\multicolumn{2}{c}{\text { Offense }} \\
\cline { 3 - 4 } \text { Defense } & D & \multicolumn{1}{c}{S} \\
\cline { 3 - 4 } & N D & \left(1-P_{C S}, P_{C S}\right) & \left(1-\frac{1}{2} P_{O S}, \frac{1}{2} P_{O S}\right) \\
\cline { 2 - 3 } & & \left(1-P_{S}, P_{S}\right) & \left(1-\frac{1}{2} P_{N S}, \frac{1}{2} P_{N S}\right) \\
\cline { 2 - 3 } & &
\end{array}
$$

\subsubsection{Analysis}

Assuming $P_{O S}>P_{N S}>P_{S}>P_{C S}$, there is no obvious pure or mixed Nash equilibrium.

If $P_{C S}>\frac{1}{2} P_{O S}$ and $P_{S}>\frac{1}{2} P_{N S}, S$ strictly dominates $N S$. Hence there is a pure strategy Nash equilibrium $(D, S)$.

If $P_{C S}<\frac{1}{2} P_{O S}$ and $P_{S}<\frac{1}{2} P_{N S}, N S$ strictly dominates $S$. Hence there is a pure strategy Nash equilibrium (ND, NS).

The following analysis of mixed Nash equilibrium is hence based on the assumption that $P_{C S}<\frac{1}{2} P_{O S}$ and $P_{S}>\frac{1}{2} P_{N S}$. It is more sensible to assume this case instead of $P_{C S}>\frac{1}{2} P_{O S}$ and $P_{S}<\frac{1}{2} P_{N S}$ because in reality, the difference between $P_{C S}$ and $P_{O S}$ is usually larger than that between $P_{S}$ and $P_{N S}$.

Proceeding to compute $P_{1}$, the equilibrium probability the offensive team lets the star player execute the play:

$$
\mathbb{E}[D]=\mathbb{E}[N D]
$$




$$
\begin{gathered}
\left(1-P_{C S}\right) P_{1}+\left(1-\frac{1}{2} P_{O S}\right)\left(1-P_{1}\right)=\left(1-P_{S}\right) P_{1}+\left(1-\frac{1}{2} P_{N S}\right)\left(1-P_{1}\right) \\
P_{1}=\frac{P_{O S}-P_{N S}}{2 P_{S}+P_{O S}-2 P_{C S}-P_{N S}} \\
\frac{\partial P_{1}}{\partial P_{S}}=\frac{2\left(P_{N S}-P_{O S}\right)}{\left(P_{S}+P_{O S}-P_{C S}-P_{N S}\right)^{2}}<0 \\
\frac{\partial P_{1}}{\partial P_{O S}}=\frac{2\left(P_{S}-P_{C S}\right)}{\left(P_{S}+P_{O S}-P_{C S}-P_{N S}\right)^{2}}>0 \\
\frac{\partial P_{1}}{\partial P_{C S}}=\frac{2\left(P_{O S}-P_{N S}\right)}{\left(P_{S}+P_{O S}-P_{C S}-P_{N S}\right)^{2}}>0 \\
\frac{\partial P_{1}}{\partial P_{N S}}=\frac{2\left(P_{C S}-P_{S}\right)}{\left(P_{S}+P_{O S}-P_{C S}-P_{N S}\right)^{2}}<0
\end{gathered}
$$

The interpretation of the partial derivatives is the same as in Case $\mathbf{A}$.

Solving for $P_{2}$, the equilibrium probability of the defensive team double-teaming the star player on the offensive team:

$$
\begin{gathered}
\mathbb{E}[S]=\mathbb{E}[N S] \\
P_{C S} P_{2}+P_{S}\left(1-P_{2}\right)=\frac{1}{2} P_{O S} P_{2}+\frac{1}{2} P_{N S}\left(1-P_{2}\right) \\
P_{2}=\frac{2 P_{S}-P_{N S}}{2 P_{S}+P_{O S}-2 P_{C S}-P_{N S}} \\
\frac{\partial P_{2}}{\partial P_{S}}=\frac{2\left(P_{O S}-2 P_{C S}\right)}{\left(2 P_{S}+P_{O S}-2 P_{C S}-P_{N S}\right)^{2}}>0 \\
\frac{\partial P_{2}}{\partial P_{O S}}=\frac{P_{N S}-2 P_{S}}{\left(2 P_{S}+P_{O S}-2 P_{C S}-P_{N S}\right)^{2}}<0 \\
\frac{\partial P_{2}}{\partial P_{C S}}=\frac{2\left(2 P_{S}-P_{N S}\right)}{\left(2 P_{S}+P_{O S}-2 P_{C S}-P_{N S}\right)^{2}}>0 \\
\frac{\partial P_{2}}{\partial P_{N S}}=\frac{2 P_{C S}-P_{O S}}{\left(2 P_{S}+P_{O S}-2 P_{C S}-P_{N S}\right)^{2}}<0
\end{gathered}
$$

The analysis is the same as in Case $\mathbf{A}$.

\section{Case C: Interior Star Player, Down by 3 Points}

This case is a trivial one as the offense is forced to let non-star players to shoot a 3-pointer. Knowing this, the defensive team will only play $N D$. Therefore, there is a pure strategy Nash equilibrium $(N S, N D)$.

\section{Case D: Interior Star Player, Down by 2 Points}

In this section, we will evaluate the case where the team has an interior star player and is down by 2 points.

\subsection{Case D1: Non-Star Player to Shoot Three-Point Shots}

This case usually happens when the team's first offensive choice is a center or power forward, but they also have decent perimeter shooters as role players. An example is the 2018-2019 Philadelphia 76ers where the star scorer is Joel Embid who is good at scoring closer to the basketball but lacks 3-pointer shooting ability; however, this is complemented by consistent shooters on the team like J.J. Redick.

The game tree is presented in Appendix C. The table of expected payoff is shown in Table 5 
Table 5. Expected payoff for Case D1

$$
\begin{array}{cc|c|c|}
\multicolumn{4}{c}{\text { Offense }} \\
\cline { 3 - 4 } \text { Defense } & D & \multicolumn{2}{c}{S} \\
\cline { 3 - 4 } & N D & \left(1-\frac{1}{2} P_{C S}, \frac{1}{2} P_{C S}\right) & \left(1-P_{O S}, P_{O S}\right) \\
\cline { 3 - 4 } & & \left(1-\frac{1}{2} P_{S}, \frac{1}{2} P_{S}\right) & \left(1-P_{N S}, P_{N S}\right) \\
\cline { 3 - 4 } & &
\end{array}
$$

\subsubsection{Analysis}

Assuming $P_{S}>P_{O S}>P_{C S}>P_{N S}$, if $P_{N S}>\frac{1}{2} P_{S}, N S$ strictly dominates $S$. Hence there is a pure strategy Nash equilibrium $(N D, N S)$.

If $P_{N S}<\frac{1}{2} P_{S}$, there is no pure strategy Nash equilibrium. The following analysis of mixed Nash equilibrium is hence based on the assumption that $P_{N S}<\frac{1}{2} P_{S}$.

Solving for $P_{1}$ :

$$
\begin{gathered}
\mathbb{E}[D]=\mathbb{E}[N D] \\
\left(1-\frac{1}{2} P_{C S}\right) P_{1}+\left(1-P_{O S}\right)\left(1-P_{1}\right)=\left(1-\frac{1}{2} P_{S}\right) P_{1}+\left(1-P_{N S}\right)\left(1-P_{1}\right) \\
P_{1}=\frac{2\left(P_{O S}-P_{N S}\right)}{P_{S}+2 P_{O S}-P_{C S}-2 P_{N S}} \\
\frac{\partial P_{1}}{\partial P_{S}}=\frac{2\left(P_{N S}-P_{O S}\right)}{\left(P_{S}+2 P_{O S}-P_{C S}-2 P_{N S}\right)^{2}}<0 \\
\frac{\partial P_{1}}{\partial P_{O S}}=\frac{2\left(P_{S}-P_{C S}\right)}{\left(P_{S}+2 P_{O S}-P_{C S}-2 P_{N S}\right)^{2}}>0 \\
\frac{\partial P_{1}}{\partial P_{C S}}=\frac{2\left(P_{O S}-P_{N S}\right)}{\left(P_{S}+2 P_{O S}-P_{C S}-2 P_{N S}\right)^{2}}>0 \\
\frac{\partial P_{1}}{\partial P_{N S}}=\frac{2\left(P_{C S}-P_{S}\right)}{\left(P_{S}+2 P_{O S}-P_{C S}-2 P_{N S}\right)^{2}}<0
\end{gathered}
$$

Solving for $P_{2}$ :

$$
\begin{gathered}
\mathbb{E}[S]=\mathbb{E}[N S] \\
\frac{1}{2} P_{C S} P_{2}+\frac{1}{2} P_{S}\left(1-P_{2}\right)=P_{O S} P_{2}+P_{N S}\left(1-P_{2}\right) \\
P_{2}=\frac{P_{S}-2 P_{N S}}{P_{S}+2 P_{O S}-P_{C S}-2 P_{N S}} \\
\frac{\partial P_{2}}{\partial P_{S}}=\frac{2 P_{O S}-P_{C S}}{\left(P_{S}+2 P_{O S}-P_{C S}-2 P_{N S}\right)^{2}}>0 \\
\frac{\partial P_{2}}{\partial P_{O S}}=\frac{2\left(2 P_{N S}-P_{S}\right)}{\left(P_{S}+2 P_{O S}-P_{C S}-2 P_{N S}\right)^{2}}<0 \\
\frac{\partial P_{2}}{\partial P_{C S}}=\frac{P_{S}-2 P_{N S}}{\left(P_{S}+2 P_{O S}-P_{C S}-2 P_{N S}\right)^{2}}>0
\end{gathered}
$$




$$
\frac{\partial P_{2}}{\partial P_{N S}}=\frac{2\left(P_{C S}-2 P_{O S}\right)}{\left(P_{S}+2 P_{O S}-P_{C S}-2 P_{N S}\right)^{2}}<0
$$

\subsection{Case D2: Non-Star Player to Shoot Two-Point Shots}

The analysis of this case is the same as Case A. However, because both the star player and non-star player will attempt a two-point shot, the set of parameters is essentially different from that used in Case A.

\section{Evaluation}

In this section, data of $P_{S}$ and $P_{N S}$ are taken from 2018-2019 NBA regular season (NBA, 2020).

For perimeter star players, take $P_{S}$ as the average of three-point field goal percentage of 10 players with the highest three-point field goals made per game $P_{S}=0.390$. Assume $P_{C S}=0.150$.

For interior star players, take $P_{S}$ as the average of field goal percentage of 10 players with the highest two-point field goal made per game $P_{S}=0.559$. Assume $P_{C S}=0.350$.

For non-star players, take $P_{N S}$ as the league average field goal percentage, $P_{N S}=0.355$ for three-point shots and $P_{N S}=$ 0.461 for two-point shots. Assume that $P_{O S}=0.400$ for three-point shots and $P_{O S}=0.550$ for two-point shots.

The results are as follows $\left(P_{1}\right.$ represents the probability of the offensive team playing $S$ and $P_{2}$ represents the probability of the defensive team playing $D)$ :

Table 6. Results

\begin{tabular}{ccccccc}
\hline & Cases A & Case B1 & Case B2 & Case C & Case D1 & Case D2 \\
\hline$P_{1}$ & 0.158 & 0.158 & 0.156 & 0 & 0 & 0.299 \\
$P_{2}$ & 0.123 & 0.123 & 0.561 & 0 & 0 & 0.329 \\
\hline
\end{tabular}

Note. In Case C and Case D1, there is a pure strategy Nash equilibrium $(N D, N S)$.

In light of the prevalent notion that star players should step up and take the last shot to determine the outcome of a game, the results seem counter-intuitive as they suggest that the shooting team will only play the star player at most 3 in 10 times among all scenarios. The results also provide insights into defensive strategies as the defensive team can act based on the composition of players of the shooting team.

Nevertheless, the preceding analysis does not account for several practical considerations in a real game. Firstly, the analysis of mixed strategy Nash equilibrium is based on many repetitions of the same game, but in discrete real-game situations, the strategies are only made once (Chow et al. 2020). In an actual game, the coach is likely to choose the player who has a relatively high shot percentage in that game to take the shot instead of selecting randomly.

Secondly, the defensive ability of the leading team can also affect the defensive strategy. If the defending team has an outstanding defender, the coach might want to instruct him to defend the star player on the other team alone.

Moreover, in reality, most coaches would be risk averse and they would not risk doubling the star player in fear of the other team getting an open shot, since even if the offensive team chooses $S$ and the defensive team chooses $D$, the star player can react to the double-team defense and pass the ball to an open teammate. In this case, an open game-winning (or game-tying) shot delivered by the offensive team can cause criticism to the defensive team's coach's strategies and decision-making abilities, and hence $N D$ might be generally preferred in some cases as it puts the coach's reputation at a lower risk.

Besides, If the coach plays non-star players most of the times, many fans who come to see their favourite star players might be disappointed. This might also potentially sow discord between star players and the coach, since not playing star players in clutch times can be easily interpreted as a sign of distrust.

End-game situations are further complicated by time restriction and tremendous pressure. Star players might be better at handling defensive pressure and thus maintaining their usual field goal percentages under tighter defense. Besides, they are also expected to shoulder a greater responsibility as they are paid more. However, non-star players could be more vulnerable to aggressive defense and stress due to time constraints.

From the defensive team's point of view, when their opponent has a perimeter star player, they should double him more when the offense tends to distribute its shots more equally between two- and three-pointers (Case B2) as compared to when the offensive team has a strong tendency to shoot three-pointers (Case A and Case B1).

The opposite analysis applies when the offensive team has an interior player. When they predominantly attempt two-point 
shots (Case D2), the defensive team should double him more than when the offensive team does not have a specific preference of the type of shots (Case D1 - this is an extreme case as there is a pure strategy Nash equilibrium).

\section{Conclusion}

This paper aims to examine how different types of players on the offensive team can affect the end-game strategy. To model this strategic situation, players are divided into star (perimeter and interior) and non-star, and several assumptions are made about the two types of players. Scenarios are categorised based on the point difference and the type of star player on the trailing team. Then, mixed strategy equilibrium and comparative statics are computed in each scenario. The analysis leads to the following conclusions: when the offensive team performs better under normal defense, they should play the star player less, and vice versa; when the offensive team has a better star player, they should double him more; in the last section, the results suggest that the offensive team should let their star player execute the play at most 3 out of 10 times, which is significantly less than they actually do.

These results contribute to the existing literature of optimal end-game strategies. Although this paper omits some features in a game such as intentional fouling as a defensive strategy and the individual defensive ability of the leading team, this framework of game model can be synthesized with other strategic situations to achieve a greater complexity. Besides, the analysis provided in this paper can be easily applied to similar strategic situations in other sports, such as football.

Future investigations are necessary to address the limitations of the methodology of this paper. A more comprehensive analysis needs to incorporate contingencies and more features of the game. Statistical analysis is also required to validate the theoretical results presented in this paper. However, complete data sets to test the results of this paper are not readily available due to the difficulty in the collection of data of shots under different defensive settings. Other possible challenges include finding an exact set of criteria to classify star and non-star players.

\section{Acknowledgements}

The author is grateful to Professor Mark C. Foley and Leslie Nut-Komlah of Davidson College for the help provided in revising the paper.

\section{References}

Annis, D. H. (2006). Optimal end-game strategy in basketball. Journal of Quantitative Analysis in Sports, 2(2). https: //doi.org/10.2202/1559-0410.1030

Ashour, M. A. H., Al-Dahhan, I. A. H., \& Al-Qabily, S. M. A. (2019). Solving game theory problems using linear programming and genetic algorithms. Advances in Intelligent Systems and Computing Human Interaction and Emerging Technologies, 247-252. https://doi.org/10.1007/978-3-030-25629-6_39

Carter, S. M. (2019). Here's how much money the winners of this years nba finals could take home. Retrieved from https://www.cnbc.com/2019/05/30/how-much-money-the-winners-of-the-nba-finals-could-take-home.html

Charkrabarti, D., \& Dutta, R. (2019). A game theoretic approach to modelling basketball. Retrieved from https://dutta. github.io/game_theory.html

Chow, T., Miller, K., Nzima, S., \& Winder, S. (2020). Shot options. http://faculty.haas.berkeley.edu/rjmorgan/mba211/ Chow\%20Heavy\%20Industries\%20Final\%20Project.pdf

Kenter, F. H. J. (2015). An analysis of the basketball endgame: When to foul when trailing and leading. https://www.stat. berkeley.edu/ aldous/157/Papers/kenter.pdf

McCarney, D. (2018). Nine coaching changes mark start of 2018-19 season. Retrieved from https://www.nba.com/article/ 2018/10/10/new-coaches-2018-19-nba-season

NBA (2020). Nba stats. Retrieved August 20, 2020, from https://stats.nba.com//

Ni, K., \& McGarrity, J. P. (2013). Strategic play in basketball when the coach's input changes. Journal Of Business Administration Online, 12(1). https://www.atu.edu/jbao/docs/strategic_play.pdf

Palacios-Huerta, I. (2003). Professionals play minimax. Review of Economic Studies, 70(2), 395-415. https://doi.org/10. 1111/1467-937x.00249

Ruminski, S. (2012). Game Theory and Basketball by Shawn Ruminski. Retrieved from https://mindyourdecisions.com/ blog/2012/06/19/game-theory-applied-to-basketball-by-shawn-ruminski/ 
Sindik, J., \& Vidak, N. (2008). Application of game theory in describing efficacy of decision making in sportsman's tactical performance in team sports. Interdisciplinary Description of Complex Systems, 6, 53-66.

Skinner, B. (2012). The problem of shot selection in basketball. PLOS ONE, 7(1). https://doi.org/10.1371/journal.pone. 0030776

\section{Appendix A}

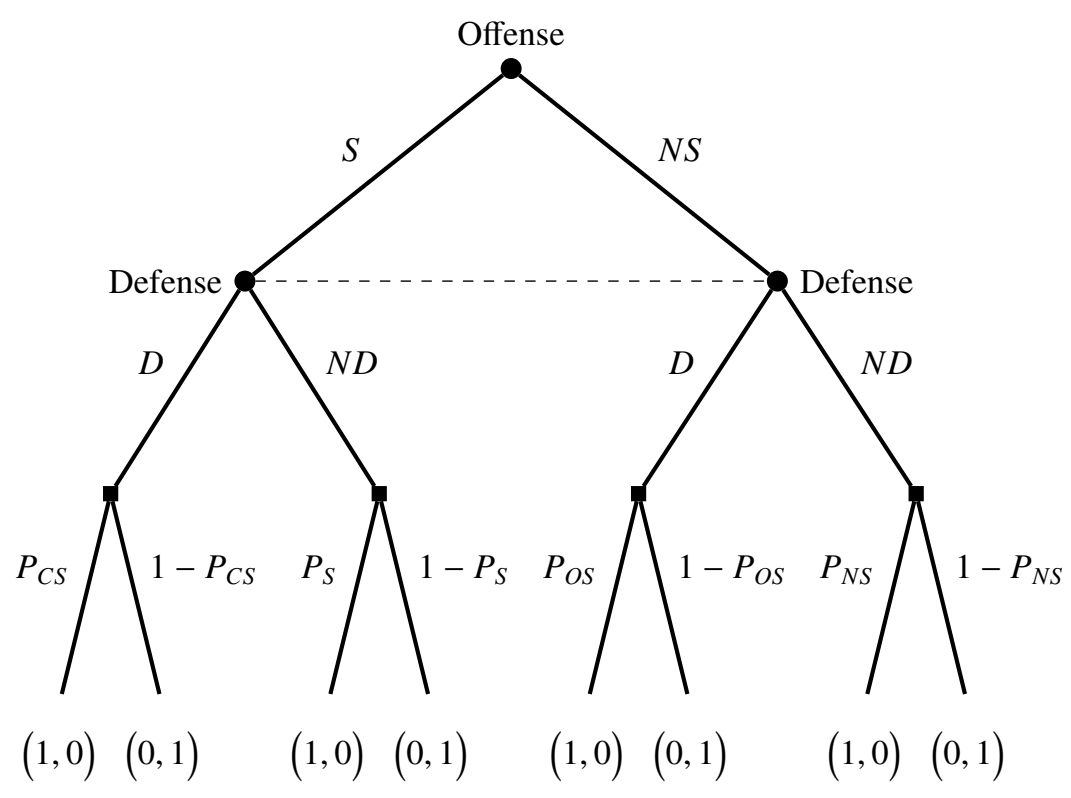

Figure 2. Game tree for Case B1

\section{Appendix B}

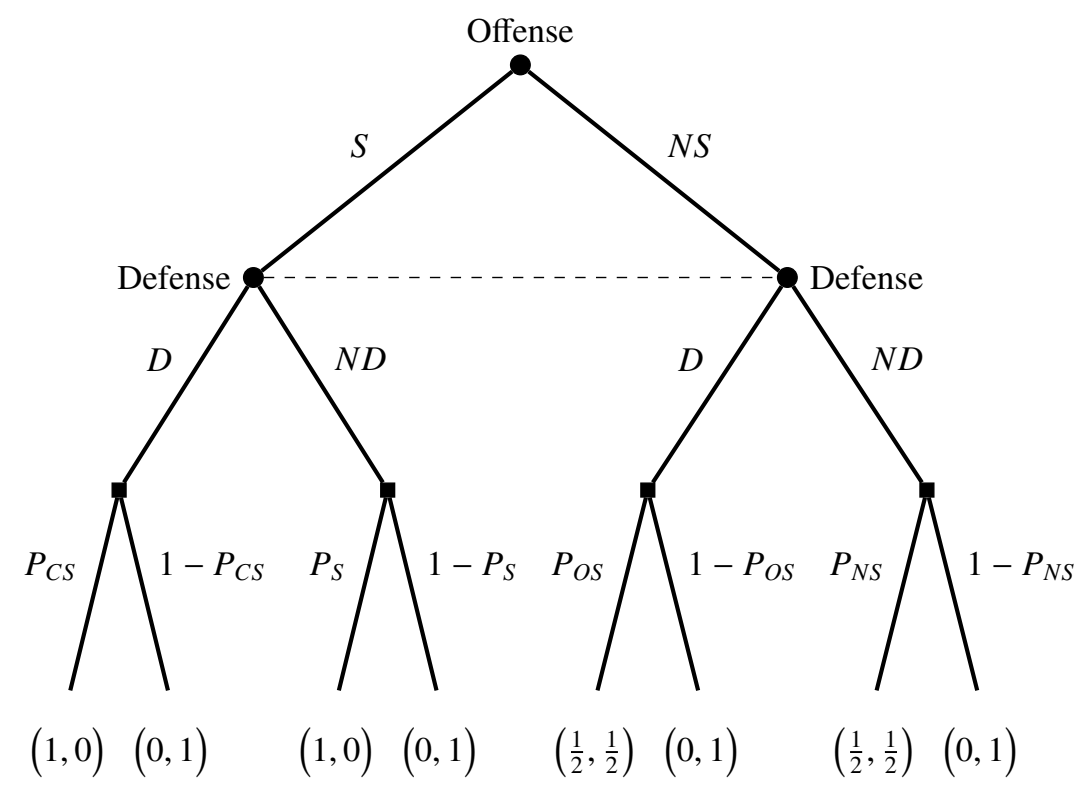

Figure 3. Game tree for Case B2 


\section{Appendix C}

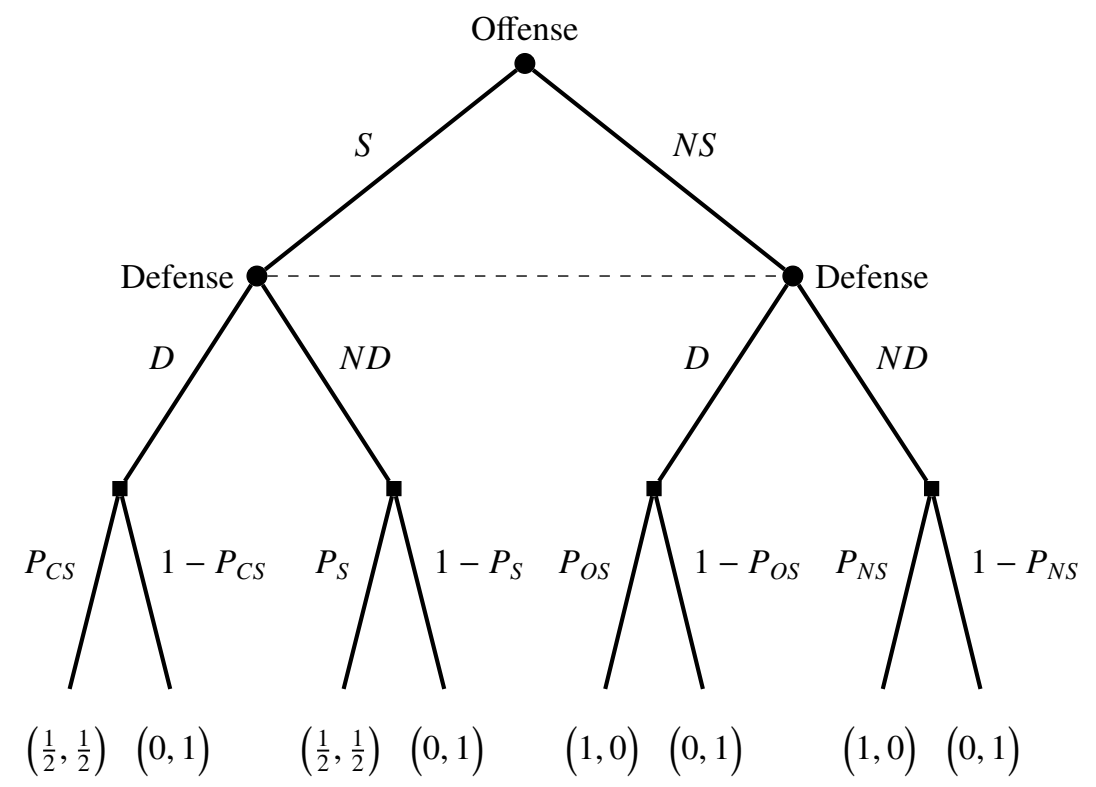

Figure 4. Game tree for Case D1

\section{Copyrights}

Copyright for this article is retained by the author(s), with first publication rights granted to the journal.

This is an open-access article distributed under the terms and conditions of the Creative Commons Attribution license (http://creativecommons.org/licenses/by/4.0/). 